\title{
Comparison of 3 absolute gravimeters based on different methods for the e-MASS project
}

\author{
Anne Louchet-Chauvet, Sébastien Merlet, Quentin Bodart, Arnaud Landragin and Franck Pereira Dos Santos, \\ Henri Baumann, \\ Giancarlo D’Agostino and Claudio Origlia
}

\begin{abstract}
We report on the comparison between three absolute gravimeters that took place in April 2010 at Laboratoire National de Métrologie et d'Essais. The three instruments (FG5\#209 from METAS, Switzerland, IMGC-02 from INRIM, Italy, and CAG from LNE-SYRTE, France) rely on different methods: optical and atomic interferometry. We discuss their differences as well as their similarities. We compare their measurements of the gravitational acceleration in 4 points of the same pillar, in the perspective of an absolute determination of $g$ for a watt balance experiment. The three instruments performed repeatable $g$ measurements, but do not agree at the level aimed for. This work calls for additional studies on systematic effects.
\end{abstract}

\section{INTRODUCTION}

The National Metrology Institutes of Switzerland (METAS), Italy (INRIM) and France (LNE) are involved in the e-MASS Euramet Joint Research Project [1]. This project aims at giving a new definition of the kilogram with the help of a watt balance, which weighs a reference mass in terms of electrical quantities [2]. In the gravimetry section of this project, two tasks have been identified: (i) determine the value of the gravitational acceleration with absolute gravimeters, and (ii) transfer the absolute value to the position of the test mass [3], [4]. The objective we pursue is to reach an accuracy at the $\mu \mathrm{Gal}$ level on both tasks. This paper reports on our efforts towards the completion of the first one. Although conventional optical gravimeters allow routine measurements of $g$ with repeatabilities of the order of a few $\mu \mathrm{Gal}$ [5], comparisons with instruments based on other technologies are desirable to confirm the accuracy of their measurements, especially in the context of the possible redefinition of SI units.

To that end, the three institutes operate three different absolute gravimeters which rely on different measurement methods. METAS operates a commercial absolute gravimeter FG5\#209 from Micro-g Lacoste, relying on the direct free-fall method. INRIM has been developing its own ballistic gravimeter IMGC-02 based on the symmetrical rise-and-fall method. LNE-SYRTE has been developing a cold atom gravimeter (CAG) based on atom interferometry, to be used with the watt balance in construction at Laboratoire National de Métrologie

A. Louchet-Chauvet, S. Merlet, Q. Bodart, A. Landragin and F. Pereira Dos Santos are with LNE-SYRTE, CNRS UMR 8630, UPMC, 61 avenue de l'Observatoire, 75014 Paris, France.

H. Baumann is with Federal Office of Metrology METAS, Lindenweg 50, CH-3003 Wabern-Bern, Switzerland.

G. D'Agostino and C. Origlia are with Istituto Nazionale di Ricerca Metrologica INRIM (formerly IMGC-CNR), Strada delle Cacce 73, 10135 Turin, Italy. et d'Essais (LNE) [6]. We report on a comparison involving these three devices, organized at LNE in a room dedicated to gravimetry, next to the watt balance room.

\section{PRESEnTAtion OF THE THREE GRAVIMETERS}

The three gravimeters involved in the comparison are national references for their respective country. All of them are based on tracking the trajectory of a free-falling test mass with a laser, using an interferometric method. However, they use different methods to measure $g$. Although they all involve vibration-rejection systems, their sensitivities are still limited by mechanical vibrations.

\section{A. Optical gravimeter FG5}

The FG5\#209 absolute gravimeter of METAS is a state-ofthe-art commercial gravimeter [7]. It is essentially a modified Mach-Zehnder interferometer, in which one arm is reflected on a free-falling corner cube. The corner cube is placed in a carriage that is lifted to the top of an evacuated dropping chamber. The carriage then accelerates downwards with an acceleration higher than $g$, to allow a $20 \mathrm{~cm}$ free fall of the corner cube. The trajectory is sampled by counting the interference fringes at the output of the interferometer. The laser source used is a HeNe laser frequency-stabilized on an iodine reference. The corner cube is then lifted again to the top of the chamber to prepare for the next measurement. To reduce the influence of ground vibrations, a reference corner cube is fixed to an active inertial reference (Super Spring) [8].

FG5 devices are used to determine the free fall acceleration in all the watt balance experiments [9] except at LNE [6].

\section{B. Optical gravimeter IMGC-02}

INRIM has been developing its own absolute optical gravimeter. The IMGC-02 is also an optical interferometer involving a iodine-stabilized HeNe laser. Unlike the FG5, it uses the symmetric rise-and-fall method: the test mass, a corner cube, is thrown vertically upwards in an evacuated chamber. A reference corner cube is fixed to the inertial mass of a long-period seismometer. The trajectories are reconstructed by sampling the fringes with a digital oscilloscope [10]. The acceleration experienced by the falling corner cube is determined by fitting a motion model to the tracked trajectory [11]. 


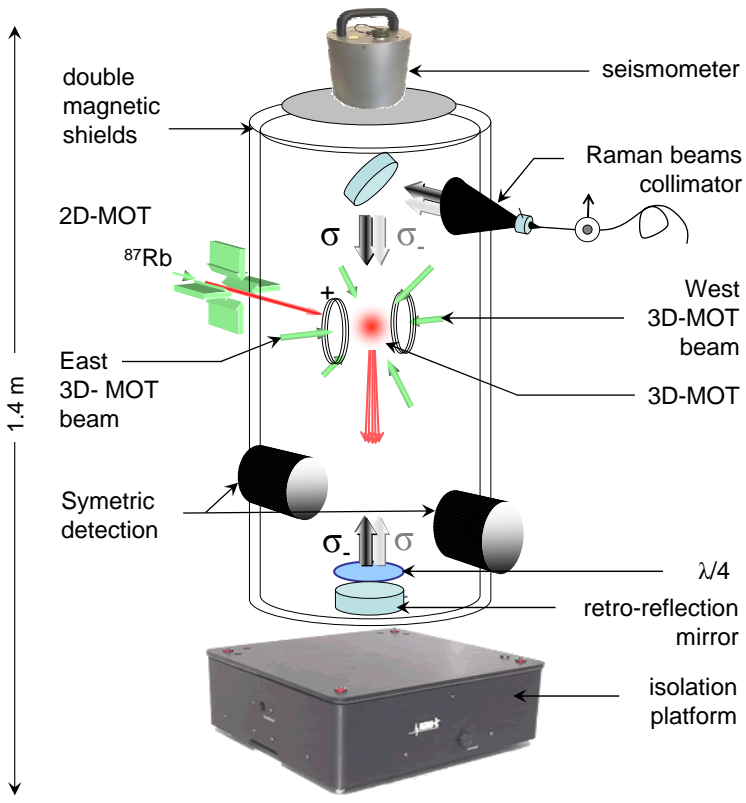

Fig. 1. Schematic of the cold atom gravimeter (CAG). A cold ${ }^{87} \mathrm{Rb}$ atomic sample is prepared by 2D- and 3D-magneto-optical trapping. After a short sub-Doppler cooling phase, where the atoms reach a temperature of $\sim 2 \mu \mathrm{K}$, the trapping beams are switched off and the atoms start their free fall in the vacuum chamber. During the free fall, a sequence of three stimulated Raman transitions is used to realize the atomic interferometer. These transitions are performed with two vertical, counter-propagating, circularly polarized laser beams, addressing the hyperfine transition of rubidium at $6.834 \mathrm{GHz}$ via two-photon excitation. The phase shift at the output of the interferometer is deduced from a symmetric fluorescence measurement of the atomic state at the bottom of the vacuum chamber [12].

\section{Atomic gravimeter $C A G$}

The cold atom gravimeter developed at LNE-SYRTE uses atom interferometry to perform a cyclic absolute measurement of $g$. At each cycle, a new cold cloud of $\mathrm{Rb}$ atoms is prepared in a UHV chamber, to be used as a test mass. During their free fall, these atoms undergo three stimulated Raman transitions that respectively separate, redirect, and recombine the atomic wave function, resulting in an atomic interferometer. The total phase shift between the two paths of this atomic interferometer depends on $g$, and scales with the square of the time interval between two consecutive Raman pulses. This gravity phase shift is cancelled by chirping the frequency difference of the two Raman beams in order to compensate the time-dependent Doppler shift. The value of $g$ is therefore derived from a frequency chirp.

A more complete description of the CAG is given in Figure 11. The vacuum chamber lies on top of a passive isolation platform. The non-filtered vibration noise is measured with a Guralp seismometer rigidly attached to the vacuum chamber, and is used to post-correct the atomic signal [13].

The device used in this comparison is an improved version of the prototype gravimeter described in [13]. The vacuum chamber is now made of titanium, in order to minimize magnetic field gradients and Eddy currents. The retroreflecting mirror for the Raman beams is placed inside the chamber, leading to reduced optical wavefront aberrations. Furthermore, the fluorescence detection is performed with a double set of

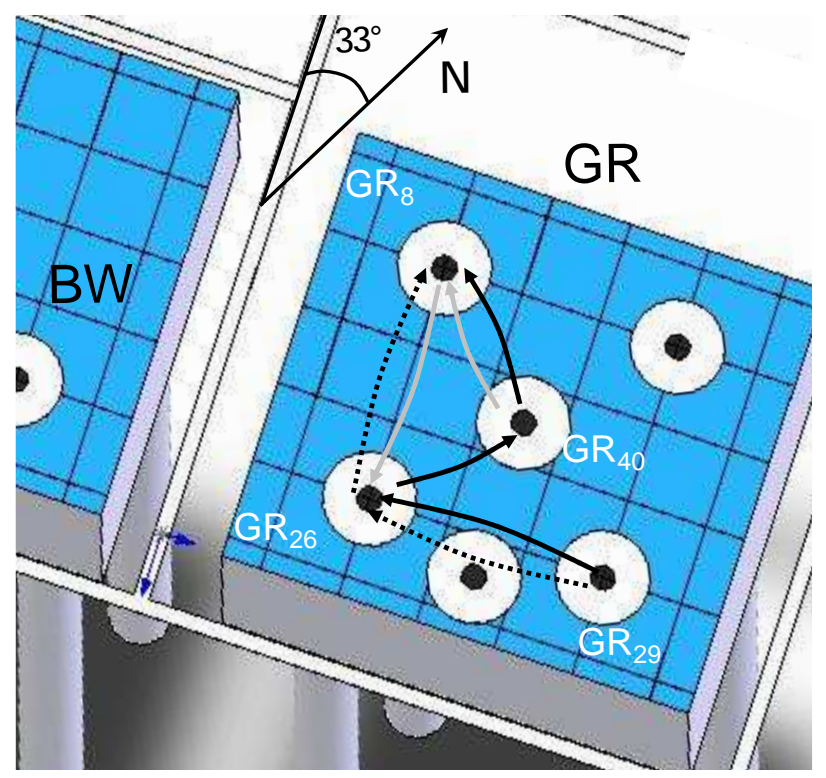

Fig. 2. Sequence of the comparison in the GR room at LNE, from April 11th 2010 (MJD=55297) to April 21st 2010 (MJD=55307). The different devices' sequences are symbolized with dashed, gray, and black arrows, for FG5\#209, CAG and IMGC-02, respectively. The LNE watt balance is located in the next room (BW), on a similar pillar.

detectors placed symmetrically at opposite sides of the atomic cloud.

Although the CAG is by far the largest of the 3 gravimeters, it is nevertheless transportable and can be moved from one point to another in a room in about 2 hours.

\section{Details ABOUT THE COMPARISON}

The comparison was carried out between April 11th and April 21st 2010 in the Laboratoire National de Métrologie et d'Essais (LNE). The three devices measured gravity in different points of the GR room next to the BW room, where the watt balance is being developed at LNE.

Figure 2 illustrates the schedule of the comparison procedure, as well as the room configuration. Gravity was measured in four different locations on the pillar, denoted GR40, GR8, GR26 and GR29. Each gravimeter measured $g$ in at least three out of these four points. Extensive relative gravimetry characterizations had previously been performed in the GR room [3]. In particular we had measured the ties between the different points as well as the vertical gravity gradients with the commercial Scintrex CG5-S105 relative gravimeter. We measured again the vertical gravity gradients right after the comparison at the four measurement points, following the same procedure, and found results in perfect agreement with the previous determination.

The $g$ values measured with the METAS FG5\#209 are given at a height of $122 \mathrm{~cm}$ above ground. Every 30 minutes, the device performs a series of 100 drops spaced by $10 \mathrm{~s}$. The IMGC-02 gravimeter performs $g$ measurements $47.2 \mathrm{~cm}$ above ground, at a rate of one throw every $30 \mathrm{~s}$, only during nighttime where the environmental noise is weaker. Concerning the $\mathrm{CAG}$, a measurement of $g$ is achieved every $0.36 \mathrm{~s}$, which 
corresponds to a repetition rate of $2.8 \mathrm{~Hz}$. The measurement height is $83.5 \mathrm{~cm}$ above ground.

For the final comparison results, the $g$ values will be given at the height of $84.25 \mathrm{~cm}$ above ground, which is the mean height of the 3 devices. The transfer of $g$ is calculated from the measured vertical gravity gradients.

\section{RESULTS}

\section{A. Gravity measurements}

Figure 3 displays the non-corrected $g$ measurements over the whole duration of the comparison, with a binning time of $150 \mathrm{~s}$ corresponding respectively to 8 drops for FG5, 5 throws for IMGC-02, and 400 drops for CAG.

For the FG5\#209 measurements, errors in the fringe counting process led to rejecting $18 \%$ of the data points lying far off from the center of the statistical distribution, otherwise fairly gaussian.

For the IMGC-02, the outliers rejection method is based on the Chauvenet criterion. The main part of the rejected data corresponds to excessive variations of the fringe visibility during the launch. This amounts to a rejection of $47 \%$ of the data points.

The cold atom gravimeter performed almost continuous $g$ measurements throughout the 10 days of the comparison. The only interruptions in the data acquisition correspond either to optical realignment and laser relocking sessions, or to the transportation of the device to another point in the room. A $3 \sigma$ rejection scheme would discard about $0.6 \%$ of the data points, i.e. of the order of what one would obtain with a normal distribution. No rejection scheme was finally applied for the CAG measurements.

The noise in the CAG measurements appears to be changing over the course of the comparison. Although we cannot rule out the possibility that the vibration noise depends on the position of the instrument in the room, it is more likely that the noise level variations are due to more or less efficient vibration isolation. The particularly noisy measurements performed on $\mathrm{MJD}=55300$ can be explained by an imperfect adjustment of the isolation platform, as well as non-optimal vibration correction parameters. Conversely, the quiet measurements performed from MJD $=55303$ to 55305 can be attributed to a combination of favorable conditions: good weather, little human activity (saturday and sunday), excellent correction of vibrations, and also relatively small power fluctuations in the Raman beams.

\section{B. Corrections to the $g$ measurements}

To get an absolute measurement of $g$, we correct the measurements from the usual environmental perturbations: polar motion, atmospheric pressure, tides and ocean loading, using different models that agree with each other. The three instruments also suffer from systematic effects, such as alignment, optical beam quality, self gravity, Coriolis effect, or reference frequency offset, for instance.

The instrument-specific corrections and corresponding uncertainties for the optical gravimeters FG5 and IMGC-02 are taken from references [7] and [14], respectively. For the CAG, we give a more complete description of the corrections that are the most delicate to evaluate, namely the Coriolis effect, optical aberrations in the Raman beams, and two-photon light shift.

The non-zero initial velocity of the atomic cloud in the EastWest direction gives rise to a bias on the gravity measurement coming from the Coriolis force. To estimate this bias we rotate the cold atom gravimeter by $180^{\circ}$ around the vertical axis. In these two configurations the contribution of the Coriolis effect has the same amplitude but opposite sign. The gravity measurements are therefore corrected with half of the difference between North and South configuration: $(-1.5 \pm 0.5) \mu \mathrm{Gal}$.

A non-plane transverse wavefront of the Raman laser beams induces a bias on the $g$ measurement [15]. This wavefront is not well known, although much closer to a plane than in the prototype version of the gravimeter [13]. The correction to $g$ due to optical aberrations is estimated by measuring the dependence of $g$ to atomic temperature, for temperatures ranging from $2 \mu \mathrm{K}$ to $10 \mu \mathrm{K}$. Indeed, the higher the temperature, the more the atomic cloud expands transversally and a larger area of the optical wavefront is probed. An unbiased $g$ value should be obtained for a non-expanding atomic cloud, corresponding to zero temperature. We therefore extrapolate the bias to $0 \mu \mathrm{K}$ and get a correction of $(0 \pm 6) \mu \mathrm{Gal}$. This effect is the main contribution to the uncertainty budget. A more thorough investigation of the optical aberrations is necessary, and will require a better control of the initial position and velocity distribution of the atomic cloud.

The two-photon light shift due to the Raman light pulses displaces the atomic levels and therefore modifies the hyperfine transition frequency [16]. Over the whole duration of the comparison, the bias on $g$ induced by the two-photon light shift varied between 8.9 and $15.5 \mu \mathrm{Gal}$, with an associated uncertainty of $0.5 \mu \mathrm{Gal}$. In order to optimize long-term stability, this effect is continuously monitored by using four interlaced successive measurement configurations [16]. Thus the corrected $g$ value is obtained from a linear combination of the four measurements, which scales down the repetition rate to $0.7 \mathrm{~Hz}$, and finally deteriorates the sensitivity of the $g$ measurement by $\sqrt{10}$.

\section{Absolute gravity measurements}

In Table $[$ and Figure 4 we show the result of the $g$ measurements performed by the 3 absolute gravimeters, transferred at $84.25 \mathrm{~cm}$ above ground. In the table we give the experimental standard deviation of the mean value $s_{g m}$ and the measurement combined uncertainty $u_{g m}$, at each gravimeter's height of measurement. We also specify $u_{t i e}$, the uncertainty due to the transfer of $g$ to the height of $84.25 \mathrm{~cm}$. In Figure 4 the total expanded uncertainty is given by $U=k \sqrt{u_{g m}^{2}+u_{t i e}^{2}}$ with $k=2$. For the 3 instruments, $u_{t i e}$ is a negligible contribution to the total uncertainty.

Without considering the IMGC-02 measurement in GR40, the gravity variations on the GR pillar measured by each device agree with the model [3] and with the ties determined with the CG5. For example, $g_{\mathrm{GR} 8}-g_{\mathrm{GR} 40}=-4.1 \mu \mathrm{Gal}$ for $\mathrm{CAG}$, in agreement with the difference of $-4.5 \mu \mathrm{Gal}$ obtained 


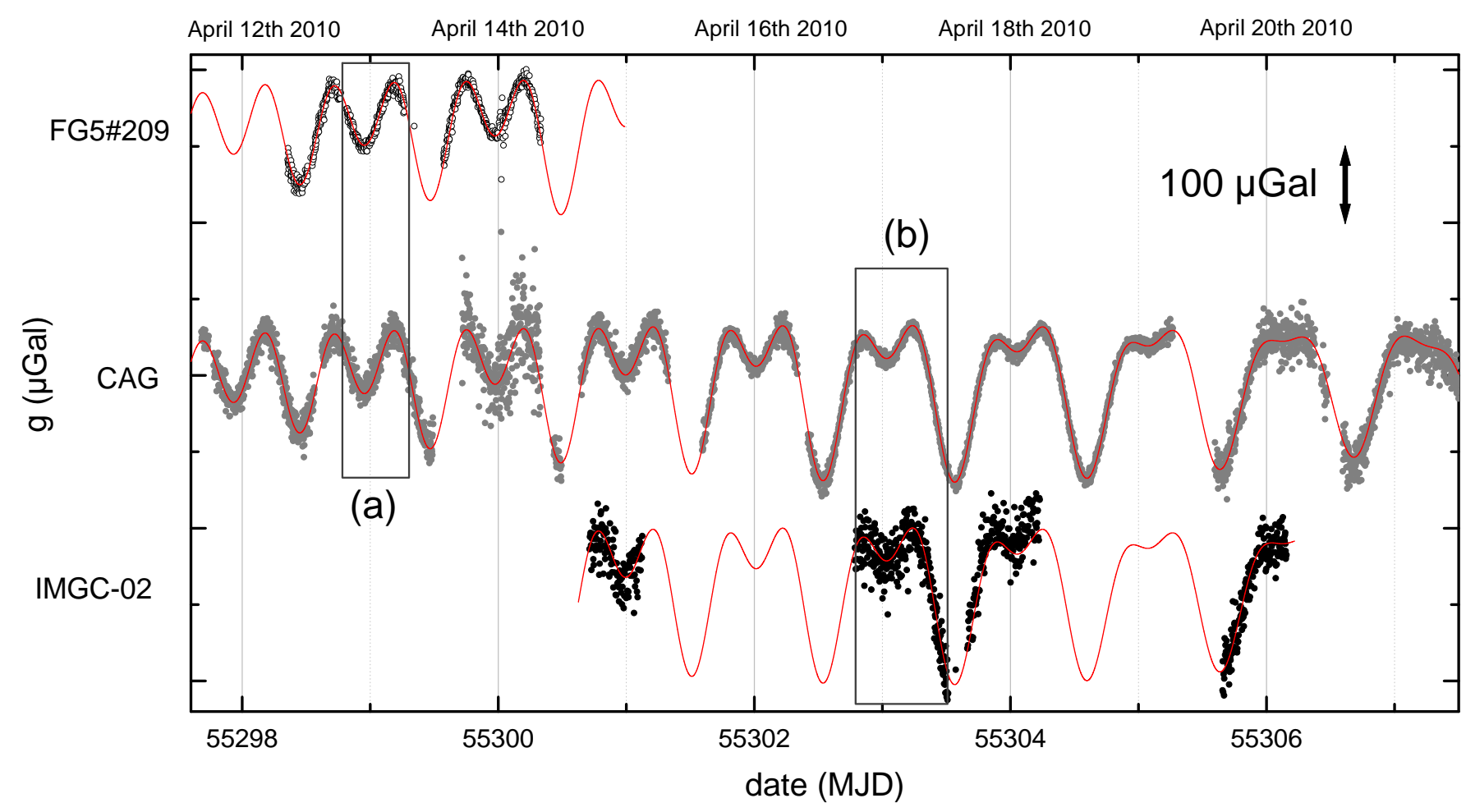

Fig. 3. $g$-measurements over the duration of the comparison, from April 11th to April 21st 2010. Open circles: FG5\#209 (bins of 8 drops); Gray circles: CAG (bins of 400 drops); Black circles: IMGC-02 (bins of 5 throws); Solid red line: tide model. The $g$ values are vertically offset between different devices for clarity. The rectangles denoted (a) and (b) indicate the time period where the Allan deviations represented in Figure 5 have been computed.

TABLE I

$g$ VALUES MEASURED 84.25CM ABOVE GROUND EXPRESSED IN $\mu \mathrm{GAL}$. $u_{t i e}$ IS THE UNCERTAINTY DUE TO THE TRANSFER OF $g$ TO THE HEIGHT OF $84.25 \mathrm{CM} ; s_{g m}$ IS THE EXPERIMENTAL STANDARD DEVIATION OF THE MEAN VALUE; $u_{g m}$ IS THE MEASUREMENT COMBINED UNCERTAINTY.

\begin{tabular}{|c|c|c|c|c|c|c|}
\hline point & MJD & gravimeter & $g$ & $u_{\text {tie }}$ & $u_{g m}$ & $s_{g m}$ \\
\hline GR40 & 55298.6 & CAG & 980890869.7 & 0.3 & 6.5 & 0.4 \\
& 55300.0 & CAG & 980890870.4 & 0.3 & 6.6 & 1.2 \\
& 55301.0 & CAG & 980890870.0 & 0.3 & 6.5 & 0.5 \\
& 55304.0 & IMGC-02 & 980890859.5 & 1.4 & 4.4 & 1.5 \\
\hline GR8 & 55300.0 & FG5\#209 & 980890854.3 & 1.4 & 2.5 & 1.5 \\
& 55301.9 & CAG & 980890865.7 & 0.3 & 6.5 & 0.3 \\
& 55303.0 & CAG & 980890866.0 & 0.3 & 6.5 & 0.2 \\
& 55304.4 & CAG & 980890865.9 & 0.3 & 6.5 & 0.2 \\
& 55305.9 & IMGC-02 & 980890843.7 & 1.4 & 4.3 & 1.4 \\
\hline GR26 & 55298.9 & FG5\#209 & 980890851.3 & 1.4 & 2.6 & 1.7 \\
& 55303.1 & IMGC-02 & 980890842.7 & 1.4 & 4.3 & 1.2 \\
& 55306.0 & CAG & 980890866.9 & 0.3 & 6.5 & 0.5 \\
& 55307.0 & CAG & 980890865.2 & 0.3 & 6.5 & 0.5 \\
\hline GR29 & 55298.4 & FG5\#209 & 980890854.2 & 1.4 & 2.3 & 1.1 \\
& 55300.9 & IMGC-02 & 980890840.8 & 1.4 & 4.6 & 2.0 \\
\hline
\end{tabular}

with the model. However, for IMGC-02, this difference is as large as $-17 \mu \mathrm{Gal}$, attributed to a laser malfunction observed while measuring on point GR40.

On the one hand, the instruments allow repeatable $g$ determinations over the duration of the comparison. On the other hand, absolute measurements obtained with the 3 instruments are not in full agreement, as shown in Figure 4 The difference between absolute measurements can reach as much as $24.2 \mu \mathrm{Gal}$ (CAG and IMGC-02, at point GR26), which is larger than the expanded uncertainty. These differences are related to systematic effects that remain to be evaluated

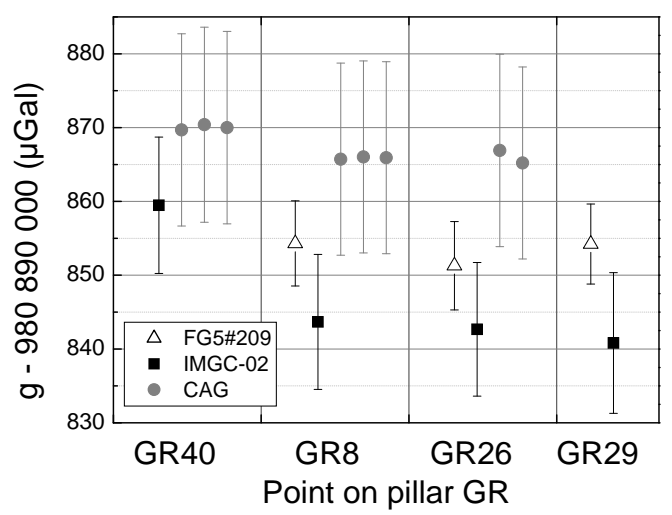

Fig. 4. Absolute $g$ measurements as given by the three gravimeters involved in the comparison. The errors bars are given with $k=2$.

more carefully, thanks to more comparisons and studies. Additionally, the results of the last International Comparison of Absolute Gravimeters (ICAG'09), where CAG, FG5\#209 and IMGC-02 were present, will bring complementary information on the repeatability of these differences.

Gravity on points GR40 and GR29 was determined during two previous comparisons. In october 2006, $g$ measurements obtained with three FG5 (\#215, \#216 and \#228) [5] showed constant differences between measurements on same points up to $10 \mu \mathrm{Gal}$. On GR29, the difference between the 2006 mean value and the FG5\#209 value obtained here is $0.4 \mu \mathrm{Gal}$.

The CAG participated in the second comparison with 


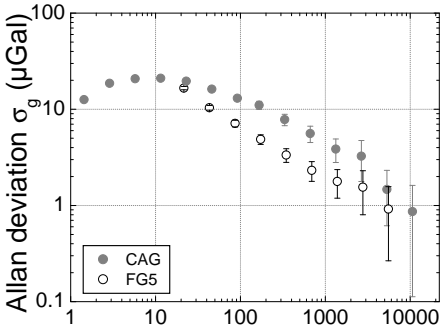

(a)

Time (s)

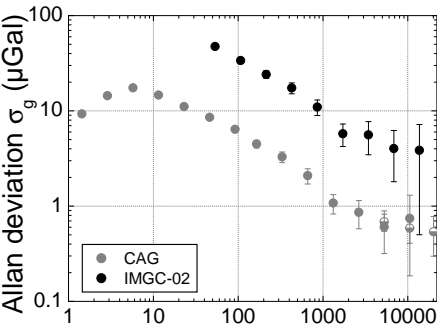

(b)

Time (s)

Fig. 5. Allan standard deviation of the corrected signals. (a) FG5 and CAG on April 13th (MJD = 55299); (b) CAG and IMGC-02 on April 17th $(\mathrm{MJD}=55303)$. In graph (b) we also plot (with semi-open circles) points corresponding to the Allan deviation for the CAG for a longer duration, which indicate that the CAG reaches a flicker floor around $(0.6 \pm 0.3) \mu \mathrm{Gal}$ after $10000 \mathrm{~s}$.

FG5\#220 [17] in october 2009. The difference between FG5\#209 obtained here and FG5\#220 obtained in 2009 is $1.6 \mu \mathrm{Gal}$ on GR29. During this last comparison, CAG performed a $g$ determination on point GR40. The difference between the CAG measurement obtained here and the one obtained in 2009 is $15 \mu \mathrm{Gal}$ at a height of $120 \mathrm{~cm}$. We believe that this discrepancy arises from relatively large -but boundedfluctuations of systematic effects in the cold atom gravimeter, rather than local variations of $g$.

\section{Sensitivity of the three gravimeters}

The sensitivity of a gravimeter is characterized by the Allan standard deviation of the corrected $g$-measurement. In Figure 5 we plot the Allan standard deviation for the corrected $g$-measurements for the three absolute gravimeters. Since we were not able to perform successful simultaneous $g$ measurements for all 3 gravimeters together, we calculate the Allan deviations over the time periods indicated by the rectangles in Figure 3. FG5 and CAG around MJD $=55299$ (a), and then CAG and IMGC-02 on MJD $=55303$ (b).

The Allan deviations of the three instruments scale as $t^{-1 / 2}$ (where $t$ is the cycle time of the measurements) which corresponds to white noise. We compare the sensitivities of the different gravimeters extrapolated to $1 \mathrm{~s}$, following this white noise behavior.

The optical interferometer IMGC-02 exhibits an equivalent sensitivity at 1 s of $\sigma_{g}=330 \mu \mathrm{Gal}$. For the FG5\#209, $\sigma_{g}=$ $70 \mu \mathrm{Gal}$. The cold atom gravimeter exhibits a sensitivity that is typically of $\sigma_{g}=140 \mu \mathrm{Gal}$ (as shown in Figure 5(a), on point GR40), but that can be as good as $60 \mu \mathrm{Gal}$ (Figure 5b), on point GR8). This variation in the noise level has already been mentioned in paragraph IV-A

\section{CONCLUSION}

In the context of the e-MASS project, we aim at determining the gravitational acceleration $g$ with an accuracy at the $\mu \mathrm{Gal}$ level, so that the contribution of the $g$ determination to the watt balance uncertainty budget is negligible. The three gravimeters that participated in the comparison reported here are based on different methods, which is relevant for an accurate determinaton of $g$.
This work is a significant step towards the achievement of the gravimetry task defined in the e-MASS project. Indeed, in this comparison, significant but repeatable differences between the 3 instruments have been measured, up to a level which is marginally compatible with the claimed uncertainties. This indicates that there are systematic effects that are not well evaluated yet. The cold atom gravimeter still requires a more complete accuracy budget determination, especially concerning the effect of optical aberrations, which we take to be responsible for long-term fluctuations of $g$ measurements. In particular, this aberration shift fluctuates due to changes in the atomic trajectories, which we plan to control better in the near future.

Additional comparisons with other absolute gravimeters based on different methods will be organized, in order to progress towards the absolute determination of $g$. The goal is to reach an agreement at the $\mu \mathrm{Gal}$ level, in the perspective of completing the gravimetric tasks of the e-MASS project.

\section{ACKNOWLEDGMENTS}

The authors would like to thank Franck Bielsa for fruitful discussions and for his invaluable help throughout the comparison.

The research within this EURAMET joint research project leading to these results has received funding from the European Community's Seventh Framework Programme, ERANET Plus, under Grant Agreement No. 217257, and from IFRAF (Institut Francilien pour les Atomes Froids). Q. B. thanks CNES for supporting his work.

\section{REFERENCES}

[1] G. Genevès, F. Villar, F. Bielsa, O. Gilbert, A. Eichenberger, H. Baumann, G. D'Agostino, S. Merlet, F. Pereira Dos Santos, P. Pinot, P. Juncar, 'The e-Mass Euramet Joint Research Project: the watt balance route towards a new definition of the kilogram", Digest of Conf. on Precision Electric Measurements (CPEM), Korea, 2010.

[2] B. P. Kibble, "Atomic masses and fundamental constants", J. H. Sanders and A. H. Wapstra (Plenum, New York, 1976), vol. 5, pp. 545-551.

[3] S. Merlet, A. Kopaev, M. Diament, G. Genevès, A. Landragin, F. Pereira Dos Santos, Micro-gravity investigations for the LNE watt balance project, Metrologia, vol. 45, pp. 265-274, 2008.

[4] H. Baumann, E. E. Klingelé, A. L. Eichenberger, P. Richard and B. Jeckelman, "Evaluation of the local value of the Earth gravity field in the context of the new definition of the kilogram", Metrologia vol. 46, pp. 178-186, 2009.

[5] S. Merlet, O. Francis, V. Palinkas, J. Kostelecky, N. Le Moigne, T. Jacobs, G. Genevès, "Absolute gravimetry measurements at LNE". Terrestrial Gravimetry Static and Mobile Measurements (TG-SMM 2007), Symp. Proc. (St Petersburg, Russia), pp. 173-174, 2007.

[6] G. Genevès, P. Gournay, A. Gosset, M. Lecollinet, F. Villar, P. Pinot, P. Juncar, A. Clairon, A. Landragin, D. Holleville, F. Pereira Dos Santos, J. David, M. Besbes, F. Alves, L. Chassagne and S. Topsu, "The BNM Watt Balance Project", IEEE Trans. Instrum. and Meas. vol. 54, pp. 850-853, 2005.

[7] T. M. Niebauer, G. S. Sasagawa, J. E. Faller, R. Hilt, F. Klopping, Metrologia, vol. 32, pp. 159-180, 1995.

[8] P. G. Nelson, "An active vibration isolation system for inertial reference and precision measurements", Rev. Sci. Instrum. vol. 62, pp. 2069-2075, 1991.

[9] A. Eichenberger, G. Genevès, P. Gournay, "Determination of the Planck constant by means of a watt balance”, Eur. Phys. J. Special Topics. 172, pp. 363-383, 2009

[10] G. D'Agostino A. Germak, S. Desogus, C. Origlia, G. Barbato, ”A method to estimate the time-position coordinates of a free-falling testmass in absolute gravimetry", Metrologia, vol. 42, pp. 233-238, 2005. 
[11] G. D'Agostino A. Germak, S. Desogus, C. Origlia, G. Barbato, "Reconstruction of the free-falling body trajectory in a rise-and-fall absolute ballistic gravimeter", Metrologia vol. 45, pp. 308-312, 2008.

[12] Ch. J. Bordé, "Atomic interferometry with internal state labelling", Phys. Lett. A, vol.140, pp. 10-12, 1989.

[13] J. Le Gouët, T. E. Mehlstäubler, J. Kim, S. Merlet, A. Clairon, A. Landragin, F. Pereira Dos Santos, "Limits to the sensitivity of a low noise compact atomic gravimeter", Appl. Phys. B, 92, pp. 133-44, 2008.

[14] G. D'Agostino, "Development and metrological characterization of a new transportable absolute gravimeter", $\mathrm{PhD}$ thesis, Politecnico di Torino, Scuola di Dottorato, 2005.

[15] J. Fils, F. Leduc, Ph. Bouyer, D. Holleville, N. Dimarcq, A. Clairon, A. Landragin, "Influence of optical aberrations in an atomic gyroscope", Eur. Phys. J. D vol. 36, pp. 257-260, 2005.

[16] A. Gauguet, T. E. Mehlstäubler, T. Lévèque, J. Le Gouët, W. Chaibi, B. Canuel, A. Clairon, F. Pereira Dos Santos, A. Landragin. "Off-resonant Raman transition impact in an atom interferometer", Phys. Rev. A vol. 78, pp. 043615, 2008.

[17] S. Merlet, Q. Bodart, N. Malossi, A. Landragin, F. Pereira Dos Santos, O. Gitlein, L. Timmen, "Comparison between two mobile absolute gravimeters: optical versus atomic gravimeters", Metrologia vol. 47, ppL9-L11, 2010. 